\title{
Develop and Validate a Metacognitive-Cognitive-Behavioral Model for Body Dysmorphic Disorder
}

\author{
Vahid Donyavi', Mehdi Rabiei², Masoud Nikfarjam ${ }^{3 *}$, Amir Mohsen Rahnejat ${ }^{4}$ \\ ${ }^{1}$ Department of Psychiatry, AJA University of Medical Science, Tehran, Iran \\ ${ }^{2}$ Department of Clinical Psychology, Baqiyatallah University of Medical Sciences, Tehran, Iran \\ ${ }^{3}$ Department of Psychiatry, Shahrekord University of Medical Science, Shahrekord, Iran \\ ${ }^{4}$ Department of Clinical Psychology, AJA University of Medical Science, Tehran, Iran \\ Email: ${ }^{*}$ nikmas140@gmail.com
}

Received 23 January 2015; accepted 3 April 2015; published 7 April 2015

Copyright (C) 2015 by authors and Scientific Research Publishing Inc.

This work is licensed under the Creative Commons Attribution International License (CC BY).

http://creativecommons.org/licenses/by/4.0/

(c) (i) Open Access

\section{Abstract}

The purpose of this study was to develop and validate a metacognitive-cognitive-behavioral model for body dysmorphic disorder. A sample of 800 participants (400 males and 400 females) was selected randomly and questionnaires were administered to them. Structure analysis was used to test the factor structure validity of the metacognitive-cognitive-behavioral model for body dysmorphic disorder. Results of the structure analysis revealed and supported a metacognitive-cognitivebehavioral model for body dysmorphic disorder. Also, the results showed that the model had the best fit to the data and was closely related to the theoretical assumptions. The model presented in this study illustrates a multidimensional approach that the model focuses on the metacognitivecognitive-behavioral dimensions; hence, the model presented in this study is a new explanatory model. The model may prompt future research into body dysmorphic disorder and facilitate clinical treatment and case formulation.

\section{Keywords}

Body Dysmorphic Disorder, Cognition, Metacognition, Behavior, Factor Analysis

\section{Introduction}

Body dysmorphic disorder (BDD) is characterized by a preoccupation with one or more perceived defects or

\footnotetext{
"Corresponding author.
}

How to cite this paper: Donyavi, V., Rabiei, M., Nikfarjam, M. and Rahnejat, A.M. (2015) Develop and Validate a Metacognitive-Cognitive-Behavioral Model for Body Dysmorphic Disorder. Open Journal of Medical Psychology, 4, 45-52. 
flaws in physical appearance that are not observable or appear slight to others, and by repetitive behaviors (e.g., mirror checking, excessive grooming, skin picking, or reassurance seeking) or mental acts (e.g., comparing one's appearance with that of other people) in response to the appearance concerns. The preoccupation causes clinically significant distress and impairment in important areas of functioning [1]. A recent Dutch study found 3\% $8 \%$ of the patients in dermatology and plastic surgery clinics of an academic hospital to be suffering from BDD [2]. Psychological and pharmacological treatments for BDD have received increasing attention in the past 10 years. Although psychological and pharmacological treatment approaches for BDD have been evaluated, the relative effectiveness of these two types of interventions has not been examined. Wiliams et al. [3] conducted a meta-analysis of randomized clinical trials and case series studies involving psychological (i.e., behavioural, cognitive-behavioural, and cognitive) or medication therapies. Their findings support the effectiveness of both types of therapy, but suggest that cognitive-behavioural treatment (CBT) may be the most useful in the long term. Also, Rabiei et al. [4] reported that MCT was an effective treatment for BDD, with effects being somewhat more pronounced on Thought-Fusion symptoms than on BDD symptoms. MCT deals with the way patients with BDD think and it assumes that the problem rests with inflexible and recurrent styles of thinking in response to negative thoughts, feelings and beliefs. In the Rabiei et al. [5] study, patients were taught that metacognitive beliefs, such as worries or rumination, were an effective desirable coping strategy, which were important factors contributing to the maintenance of BDD. However, metacognitive beliefs do not provide information that disconfirms negative beliefs or appraisals.

Metacognitive regulation refers to a broad spectrum of executive functions, such as monitoring, planning, checking, attention and detection of errors in performance [6]. Metacognitive knowledge refers to the information individuals hold about their internal states and about coping strategies that impact on them [6]. Examples of metacognitive knowledge may include beliefs concerning the significance of particular types of thoughts (e.g., "Having thought X means I am weak") and emotions (e.g., "I need to control my anxiety at all times"), and beliefs about cognitive competence (e.g. "I do not trust my problem-solving capabilities"). Examples of the information individuals hold about their own coping strategies that impact on internal states may include both positive ("Ruminating will help me find a solution") and negative ("My checking behavior is making me lose my mind") beliefs. In the metacognitive conceptualization of psychological dysfunction [7], all the above constructs interact in maintaining maladaptive behavior. The Self-Regulatory Executive function (S-REF [7]) theory was the first to conceptualize the role of metacognition in the etiology and maintenance of psychological disturbances. In this theory Wells and Matthews [7] argue that a common style of thinking across psychological disorders leads to dysfunction. They propose that psychological disturbance is maintained by a combination of perseverative thinking styles, maladaptive attentional routines, and dysfunctional behaviors. The S-REF theory has led to the development of disorder-specific models of [8], generalised anxiety disorder [7], obsessive-compulsive disorder [3], post-traumatic stress disorder [6] and body dismorphic disorder [4]. Cooper and Osman [9] suggest that patients with BDD do indeed engage in metacognitive processing in relation to their concerns with appearance. They report attempts to control, correct, appraise, and regulate their thinking in relation to images and also in relation to thoughts associated with their illness-related concerns. Thus, as suggested by Veale [10], metacognition may be an important feature of information processing in BDD and may be one way in which the symptoms of the disorder are maintained. Theoretically, therefore, it may be an important dimension to be incorporated into a cognitive model of BDD. Further research is needed into the phenomena of metacognition, including its characteristics, functions, and role in the maintenance of the distressing symptoms of BDD. Imagery has been accorded a particularly important role in the maintenance of BDD, where mental images of the self are thought to be a particularly central feature of a cognitive conceptualization [10].

Although these studies have provided some basis about cognition, metacognition, and behavior in body dysmorphic disorder and shown that there is a relationship between them, there is need for a comprehensive model to link cognition, metacognition, behavior and dimensions of body dysmorphic disorder. In the current model we propose that the personal goals, values, and thoughts that increase an individual's awareness of their appearance as well as the context of their daily experience play an important role in triggering worry about body dysmorphia. In this way our life tasks, current concerns, positive metacognitive beliefs or personal strivings can be an important catalyst for worry in the vulnerable individual. In addition we proposed that enduring schemas of low self-confidence and threat would constitute a predisposition for body dysmorphic disorder and chronic worry about body dysmorphia. The interaction of these pre-potent schemas of personal vulnerability with positive metacognitive beliefs could trigger threat-relevant intrusive thoughts or images. Intrusive thoughts are "any 
distinct, identifiable cognitive event that is unwanted, unintended, and recurrent. In the cognitive model, intrusive thoughts of uncertainty are both a cause and a consequence of threat schema activation. We would expect that these thoughts will become more frequent with sustained activation of the BDD-relevant schemas.

Wells [6] argues that positive metacognitive beliefs about worry (e.g., "Worry helps me cope") are activated early in the worry process and are central to initiating worry as a coping strategy. This results in Type I worry in which the individual focuses on the potential threat of a situation (e.g., "What if I lose my job?"). The threat and uncertainty involved in Type I worry activate negative metacognitive beliefs about worry. Beliefs about the uncontrollability and negative consequences of worry lead to Type II worry, or meta-worry, in which the individual becomes focused on trying to suppress or control worry and or the individual becomes focused on trying to mirror check, groom, or camouflage because of the associated rise in anxiety.

In this model, we believed that our view points towards negative cognitions can increase the number of our negative thought sand lead to more concentration onnegative thoughts. For example, if we have negative view points towards negative thoughts of our mind (negative metacognitive beliefs) and consider them as abnormal, we cannot go along with them in mind, and their acceptance them will be very difficult. To consider negative thoughts as abnormal and dangerous will trigger anxiety and fear. Objectivity and considering negative thoughts and worries as dangerous is a kind of cognitive distortions and removes the individual from logic and outer reality. To consider negative thoughts as dangerous and terrifying causes to metaworry. As it was mentioned above, worry may naturally occur in many people and it does not represent any disease; however, metaworry is more serious and usually leads people to disease. Meta worry pertains to considering worry as dangerous and abnormal. If individuals consider their worries as abnormal and dangerous they will become worried from the worry itself. Some instances of metaworry will illustrate the issue: Such worries happen in reality. They make people mad. They are uncontrollable, they have no end and they do not leave me alone until they defeat me. These beliefs represent metaworry and exacerbate fear and anxiety, and even natural exacerbation of physical symptoms such as increased heart rate, dry mouth, headache, numbness and numbness of the body. Meta worry exacerbates stress and anxiety, and as the mind and body cannot tolerate constant anxiety and stress, it turns to mental and behavioral strategies to calm down itself. To reduce stress and anxiety, individuals use thought control strategies, compulsive behaviors, avoidance and safety behaviors: Thought and worry control strategies include strategies applied to control and eliminate negative thoughts and worries. Of such strategies are suppression, distraction, and elimination. Using these strategies is often faced with failure since efforts to control and eliminate negative cognitions often increase rather than decrease the amount of negative cognition. Thoughts and worries often automatically enter into our minds, i.e., they are not under voluntary control and that attempt to control them will usually be subject to failure. Compulsive, avoidance and safety behaviors such as over-examination of a disease and reiteration of the related examinations, reassurance about their health, avoidance from suspected pathogenic situations and exposure to suspected pathogenic situations and even, taking drugs may temporarily bring about relaxed and satisfied feeling but it causes the sustainability of this vicious cycle in a long run.

The main aim of this study was to develop a metacognitive-cognitive-behavioral model for body dysmorphic disorder and to determine its validity.

\section{Method}

\subsection{Participants}

The data for the Iranian sample were collected in 2014. The Iranian sample consisted of 800 participants (400 male and 400 female) attending medical and psychology clinics in Isfahan, Tehran, Iran. Participants ranged in age from 15 to 62 years $(M=30.11$; S.D. $=4.77)$. Multi-stage cluster sampling method was used to select the sample. Participants' characteristics are presented in Table 1.

\subsection{Measures}

The assessment tools in this study were the Yale-Brown Obsessive Compulsive Scale Modified for BDD, Body Dysmorphic Metacognitive Scale, Dysfunctional Attitude Scale form A, Cognitive Distortion Scale and ATQ and Compulsive behaviors spectrum scale.

\subsubsection{Body Dysmorphic Metacognitive Scale (BDMCS; Rabiei) [5]}

This measure consists of 25 items used to assess metacognitive errors or distortions related to body dysmorphic 


\begin{tabular}{lc} 
Table 1. Participants characteristics $(\mathrm{N}=800)$. \\
\hline Gender [n (\%)] & 400 \\
Women & 400 \\
Men & $30.11(4.44)$ \\
Age in years [mean (S.D.)] & $15-62$ \\
Min-max & \\
Occupational status (n) & 200 \\
Working full-time & 300 \\
Student & 80 \\
Part-time work & 120 \\
Unemployed & 100 \\
Retired & \\
Education & 50 \\
Primary school & 600 \\
High school & 150 \\
University & \\
Psychotropic medication (n) & \\
SSRI & 40 \\
SNRI & 45 \\
Benzodiazepines & 111 \\
Antipsychotics & \\
Psychiatry disease duration (yr) & \\
Mean length (S.D.) & \\
Min-max & \\
\hline & \\
&
\end{tabular}

disorder. In order to obtain adequate face validity, a pool of items were used to construct BDMS from the data obtained in an earlier study (Rabiei et al., 2012) and transcripts of therapy sessions. The five factors identified (accounting for $83.8 \%$ of variance) reflected the following domains: 1) metacognitive control strategies; 2) thought-fusion; 3) positive metacognitive beliefs about emotional self-regulation; 4) positive metacognitive beliefs about cognitive self-regulation; 5) negative metacognitive beliefs about cognitive harm and uncontrollability. The convergent validity was supported by testing correlations between the BDMCS and the Yale-Brown Obsessive Compulsive Scale modified for BDD and TFI. The internal consistencies of the subscales were moderate to high, with Cronbach's alpha values ranging from 0.83 to 0.93 . Responses to each item were required on a 4-point rating scale as follows: 1 (do not agree), 2 (agree slightly), 3 (agree moderately) and 4 (agree very much) [5].

\subsubsection{Yale-Brown Obsessive Compulsive Scale Modified for Body Dysmorphic Disorder (BDD-YBOCS) [11]}

This is a reliable and valid 12-item semi-structured clinician administered instrument that evaluates current BDD severity. It assesses BDD-related preoccupations, repetitive behaviors, insight, and avoidance. The reliability and validity of the BDD-YBOCS Farsi version version was demonstrated by Rabiei et al. [4] in both healthy and clinical samples. The alpha coefficients ranged from 0.78 to 0.93 for the BDD-YBOCS total score and for its subscales (preoccupations, repetitive behaviors).

\subsubsection{The Dysfunctional Attitude Scale Form A [12]}

This is a self-report scale designed to measure the presence and intensity of dysfunctional attitudes. The DAS-A consists of 40 items and each item consists of a statement and a 7-point Likert scale ( 7 = fully agree; 1 = fully disagree). Ten items are reversely coded $(2,6,12,17,24,29,30,35,37$ and 40). The total score is the sum of the 
40-items with a range of $40-280$. The higher the score, the more dysfunctional attitudes an individual possesses [12]. Internal consistency, test-retest reliability, and average item-total correlations of the DAS-A were satisfactory in different samples [13].

\subsubsection{Cognitive Distortion Scale (CDS) [14]}

This is a self-report scale assessing five dimensions of cognitive distortions: self-criticism (SC), self-blame (SB), helplessness (HLP), hopelessness (HOP), and preoccupation with danger (PWD). This scale contains 40 items, with eight items in each dimension. Each item is rated on a 5-point Likert scale, from 1 (never) to 5 (very often). The total score for the CDS is between 40 and 200 and for each dimension the total score is between 8 and 40 . High scores indicate high cognitive distortion. The reliability of CDS for the Farsi version was $\alpha=0.97$ [14].

\subsubsection{The Automatic thoughts Questionnaire (ATQ) [15]}

The ATQ is a 30-item questionnaire developed to identify and assess the frequency of automatic negative selfstatements. Respondents are presented with 30 negative thoughts about the self (e.g., I'm a loser. My life is a mess) and asked to indicate the extent to which they have experienced these thoughts over the past week. Each item is a negative thought and the respondent is to rate how often the thought has surfaced in the past week on a scale from 1 (not at all) to 5 (all the time). ATQ-Persian had excellent internal consistency (Cronbach's alpha = $0.96)$, test-retest reliability $(r=0.84)$ and correlation with the Beck Depression Inventory $(r=0.77)[16]$.

\subsubsection{Compulsive Behaviors Spectrum Scale}

The scale is an 8-item questionnaire developed to identify and assess the frequency of compulsive behaviors. Respondents are presented with 8 compulsive behaviors about the self and asked to indicate the extent to which they have experienced these compulsive behaviors over the past week. Each item is a compulsive behaviors and the respondent is to rate how often the compulsive behaviors has surfaced in the past week on a scale from 1 (not at all) to 5 (all the time). CBSS-Persian had excellent internal consistency (Cronbach's alpha $=0.95)$, testretest reliability $(r=0.90)$ and correlation with the BDD-YBOCS $(\mathrm{r}=0.71)[17]$.

\subsection{Data Analysis}

In order to examine the factor structure of the metacognitive-cognitive-behavioral model we conducted Structural Equation Modeling. For these analyses the Structural Equation Modeling program AMOS 5 was used. Values of the Goodness of Fit Index (GFI) and the Adjusted Goodness of Fit Index (AGFI) close to 1 represent a good fit. Values of the Root Mean Square Residual (RMR) and Standardized Root Mean Square Residual (SRMR) below .05 represent a good fit, and values less than .08 represent an acceptable fit.

\section{Results}

\section{The Factor Structure of the Metacognitive-Cognitive-Behavioral Model}

The factor structure of the metacognitive-cognitive-behavioral model was examined by means of CFAs. Figure 1 shows the model structure which we finally obtained.

Through this analysis, we find out the structure among BDD variables that could be explained to define the metacognitive cognitive-behavioral model. There are significant paths to cognition, significant paths to sense of metacognition and significant paths to behavior of respondents. BDD is treated as observed, endogenous variable since we assume that it might be influenced by cognition, metacognition and behavior. All hypothesized paths were supported, all $p<0.05$, there was a direct and significant effect of core beliefs on cognitive distortion, standardized estimate $=0.49(\mathrm{p}<0.01)$, there was a direct and significant effect of positive metacognitive beliefs on cognitive distortion, standardized estimate $=0.17(\mathrm{p}<0.05)$, there was a direct and significant effect of negative thought on meta worry, standardized estimate $=0.51(\mathrm{p}<0.01)$. The standardized indirect effect of negative thought on compulsive behaviors through metaworry was $=0.25(\mathrm{p}<0.01)$, the standardized indirect effect of metaworry on BDD through compulsive behaviors was $=0.19(\mathrm{p}<0.05)$.

Findings, reported in Figure 1 and Table 2, demonstrated that the model had overall fit to the data, and is closely related to the theoretical assumptions of the metacognitive-cognitive-behavioral model.

We conduct an SEM analysis on the correlation between independent variables and to understand the indirect 


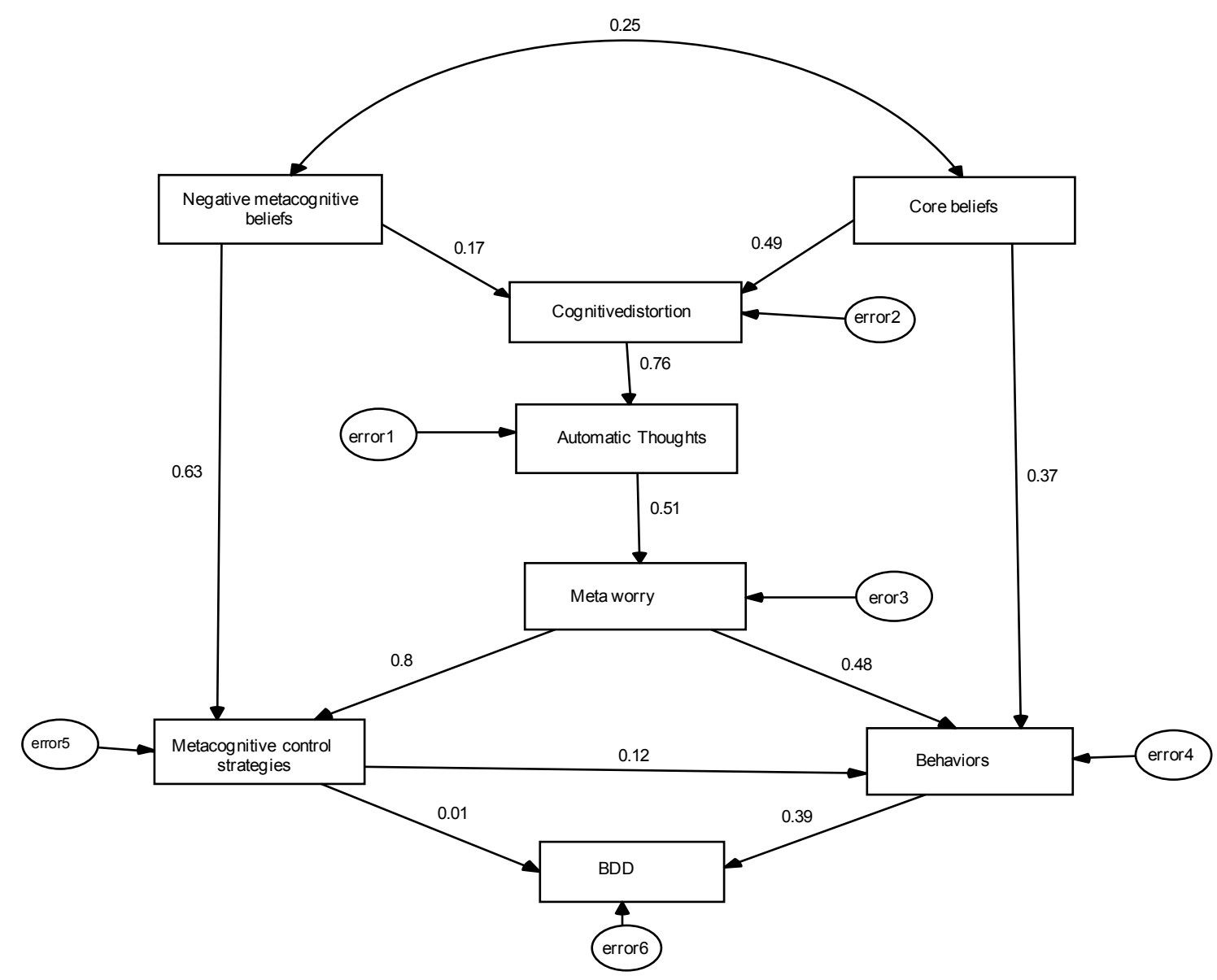

Figure 1. The factor structure of the metacognitive-cognitive-behavioral model for body dysmorphic disorder.

Table 2. Model fit indices for the model of the metacognitive-cognitive-behavioral for body dysmorphic disorder.

\begin{tabular}{ccccccc}
\hline Model & N & GFI & AGFI & RMR & RMSEA & AIC \\
\hline & 800 & 0.98 & 0.99 & 0.01 & 0.02 & 15.36 \\
\hline
\end{tabular}

Note: N: Number of Participants; GFI: Goodness of Fit Index; AGFI: Adjusted Goodness-of-Fit Index; RMR: Root Mean Square Residual; SRMR: Standardized Root Mean Square Residual; AIC: The Akaike Information Criterion.

effects. These allow us to account for correlation and distinguish direct and indirect effects of our exogenous and endogenous variables on sense of community. We have investigated several model structures and find that the model presented in Figure 1 provides the best model fit. The model fit can be considered "good" in terms of goodness of fit $(\mathrm{CMIN} / \mathrm{DF}=1.27, \mathrm{GFI}=0.98, \mathrm{AGFI}=0.99$ and $\mathrm{RMSE} 0.02)$.

\section{Discussion}

The intent of this study was to develop and validate metacognitive-cognitive-behavioral model for body dysmorphic disorder.

Findings of this study revealed that the metacognitive-cognitive-behavioral model for body dysmorphic disorder had a clear factor structure, congruent with its theoretical conceptualization (see Figure 1 and Table 2).

The higher positive correlation and significance of this model and its factors with BDD reflect the acceptable validity of this model. This finding may be helpful to derive and illustrate the role of metacognitive beliefs about BDD together with other relevant cognitive-behavioral constructs in a given episode. The distorted and faulty appraisal evident in pathological worry shares more similarities than differences with how individuals appraise other types of unwanted repetitive thoughts such as obsessions or depressive rumination. However, there is emerg- 
ing evidence that certain metacognitive processes may be especially critical to the persistence of worry. A tendency to catastrophize, to believe that negative outcomes are likely to occur and will lead to significant negative effects in one's life, and to perceive worry itself as a highly uncontrollable, disturbing, and dangerous process are metacognitive appraisals that are likely to contribute to an escalation of the worry process. Although empirical research relevant to this model is still preliminary, these early findings are encouraging for further exploration of the role of metacognitive processing in BDD.

Given that individuals with BDD tend to appraise their worrisome thoughts as disturbing and associated with a greater likelihood of negative outcomes, this model is a natural extension of the previous hypothesis. According to the cognitive model illustrated in the previous hypothesis, we predict that unsuccessful and futile efforts to control or suppress worry will paradoxically contribute to its persistence, in accord with Wegner's ironic process theory of suppression [6] [18]. As predicted by model of the study, researchers have consistently found that BDD is characterized by a heightened subjective experience of worry as an uncontrollable process and any efforts at control prove futile and unproductive. Despite their acknowledged inability to control worry, it is interesting that individuals with BDD are highly invested in continuing with their efforts toward gaining control over worry and unwanted repetitive thoughts [4] [5] [9].

The present results are preliminary in nature. Clearly, future studies are required to further establish the psychometric properties of the model. In particular, it would be necessary to determine the structure and reliability over time and with other samples. In addition, studies are required to examine the sensitivity of the model to treatment effects and recovery. The role of high levels of positive metacognitive beliefs in predisposing individuals to engage in BDD behaviors, and of negative metacognitive beliefs in maintaining problematic BDD behaviors can also be investigated through longitudinal designs. There are also limitations in this study, meaning that the results need to be interpreted with some caution. The use of a non-clinical sample may limit the generalizability of these findings to clinical populations as well as to populations that are diverse in age, socioeconomic status, and other demographic variables. The use of a non-clinical sample might also result in inflation of the associations among latent variables due to floor effects that could result from low levels of symptomology in most participants. Ultimately, only self-report measures were included for each construct. Future studies using multiple measures of each construct and with different response formats (e.g., clinician-rated, self-report, and interview) may help improve these findings.

Despite these limitations, we believe that this model may be useful in providing a step towards the development of the metacognitive-cognitive-behavioral conceptualization of BDD.

\section{References}

[1] Van Ameringen, M., Patterson, B. and Simpson, W. (2014) DSM-5 Obsessive-Compulsive and Related Disorders: Clinical Implications of New Criteria. Depress and Anxiety, 31, 487-493. http://dx.doi.org/10.1002/da.22259

[2] Vulink, N.C., Sigurdsson, V., Kon, M., Bruijnzeel-Koomen, C.A., Westenberg, H.G. and Denys, D. (2006) Body Dysmorphic Disorder in 3\% - 8\% of Patients in Outpatient Dermatology and Plastic Surgery Clinics. Ned Tijdschr Geneeskd, 150, 97-100. [Vulink, N.C., Sigurdsson, V., Kon, M., Bruijnzeel-Koomen, C.A., Westenberg, H.G. and Denys, D. (2006) Stoornis in de lichaamsbeleving bij 3\% - 8\% van de patienten op de poliklinieken Dermatologie en Plastische Chirurgie. Ned Tijdschr Geneeskd, 150, 97-100.]

[3] Williams, J., Hadjistavropoulos, T. and Sharpe, D. (2006) A Meta-Analysis of Psychological and Pharmacological Treatments for Body Dysmorphic Disorder. Behaviour Research and Therapy, 44, 99-111. http://dx.doi.org/10.1016/j.brat.2004.12.006

[4] Rabiei, M., Mulkens, S., Kalantari, M., Molavi, H. and Bahrami, F. (2012) Metacognitive Therapy for Body Dysmorphic Disorder Patients in Iran: Acceptability and Proof of Concept. Journal of Behavior Therapy and Experimental Psychiatry, 43, 724-729. http://dx.doi.org/10.1016/j.jbtep.2011.09.013

[5] Rabiei, M., Salahian, A., Bahrami, F. and Palahang H. (2011) Construction and Standardization of the Body Dysmorphic Metacognition Questionnaire. Journal of Mazandaran University of Medical Sciences, 21, 43-52.

[6] Wells, A. (2000) Emotional Disorders and Metacognition: Innovative Cognitive Therapy. John Wiley \& Sons, Chichester, New York, $236 \mathrm{p}$.

[7] Wells, A. and Matthews, G. (1996) Modelling Cognition in Emotional Disorder: The S-REF Model. Behaviour Research and Therapy, 34, 881-888. http://dx.doi.org/10.1016/S0005-7967(96)00050-2

[8] Papageorgiou, C. and Wells, A. (2003) An Empirical Test of a Clinical Metacognitive Model of Rumination and Depression. Cognitive Therapy and Research, 27, 261-273. http://dx.doi.org/10.1023/A:1023962332399 
[9] Cooper, M. and Osman, S. (2007) Metacognition in Body Dysmorphic Disorder-A Preliminary Exploration. Journal of Cognitive Psychotherapy, 21, 148-155. http://dx.doi.org/10.1891/088983907780851568

[10] Veale, D. (2004) Advances in a Cognitive Behavioural Model of Body Dysmorphic Disorder. Body Image, 1, 113-125. http://dx.doi.org/10.1016/S1740-1445(03)00009-3

[11] Hollander, E. and Benzaquen, S.D. (1997) The Obsessive-Compulsive Spectrum Disorders. International Review of Psychiatry, 9, 99-109. http://dx.doi.org/10.1080/09540269775628

[12] Weissman, A. and Beck, A. (1978) Development and Validation of the Dysfunctional Attitude Scale: A Preliminary Investigation. Paper Presented at the Annual Meeting of the American Educational Research Association, Toronto, Ontario.

[13] Ebrahimi, A., Samouei, R., Mousavii, S.G. and Bornamanesh, A.R. (2013) Development and Validation of 26-Item Dysfunctional Attitude Scale. Asia-Pacific Psychiatry, 5, 101-107. http://dx.doi.org/10.1111/appy.12020

[14] Briere, J. (2000) The Cognitive Distortion Scale Professional Manual. Psychological Assessment Resources, Odessa.

[15] Hollon, S. and Kendall, P. (1980) Cognitive Self-Statements in Depression: Development of an Automatic Thoughts Questionnaire. Cognitive Therapy and Research, 4, 383-395. http://dx.doi.org/10.1007/BF01178214

[16] Ghassemzadeh, H., Mojtabai, R., Karamghadiri, N. and Ebrahimkhani, N. (2006) Psychometric Properties of a PersianLanguage Version of the Automatic Thoughts Questionnaire: ATQ-Persian. The International Journal of Social Psychiatry, 52, 127-137. http://dx.doi.org/10.1177/0020764006062095

[17] Rabiei, M., Khormdel, K., Kalantari, K. and Molavi, H. (2010) Validity of the Yale-Brown Obsessive Compulsive Scale Modified for Body Dysmorphic Disorder (BDD) in Students of the University of Isfahan. Iranian Journal of Psychiatry and Clinical Psychology, 15, 343-350.

[18] Cooper, M. (2003) Emotional Disorders and Metacognition: Innovative Cognitive Therapy. British Journal of Clinical Psychology, 42, 105-106.

[19] Donyavi, V., Rabiei, M., Nikfarjam, M. and Nezhady, BM. (2015) Body Image and Meta-Worry as Mediators of Body Dysmorphic Disorder. International Journal of Clinical Medicine, 6, 170-176. 